\title{
Design of a graphical programming based Interactive and Computerized Eye Exercise Application
}

\author{
Banu Rekha B \\ $\{$ *bbr.bme@psgtech.ac.in $\}$ \\ Department of Biomedical Engineering, PSG College of Technology, Tamil nadu, Coimbatore-641004, \\ India $^{1}$
}

\begin{abstract}
Vision is the prominent sense of human beings and having a healthy vision becomes a prime requirement for the competitive world in modern days. Due to long time usage of computers and televisions, eye related disorders are on the rise nowadays. Physicians advise simple eye exercises like rotation and diagonal movement of eyeballs to avoid those disorders. This work is an effort to develop an interactive eye exercise regime. An object is made to move according to specific patterns on the computer screen and the eye movements of the user are recorded online. This video is then used to compare the pattern movement and eye movement and graphical result is provided to the user. This feedback may help the user in faithfully following the patterns on screen, thus completing the eye exercise in a simple but effective way.
\end{abstract}

Keywords: Graphical programming, Human computer interface, Eye exercise, Eyeball tracking, Video processing

\section{Introduction}

In this modern era, every individual has awareness about their body fitness and they strive to maintain it. But, due to usage of computers in every field, it is very much essential to take care of our eyes. Having a healthy vision is a necessity and not a luxury. Still, the society takes it light unlike other sensory organs until the vision begins to degrade or fail. Working with computers for a long duration has reported increase in eye related disorders, both in adults and children. The ciliary body, which is the eye muscle, gets strained if it is kept at a single position for a long time. Physicians advise simple eye exercises like top-bottom, left-right and diagonal direction movement of eyeballs regularly to avoid such problems. Moving our eyes in all directions without turning the head can help strengthen the eye muscles, prevent eyestrain and improve eyesight. The basic eye exercises are palming, sunning, swinging and vision shifting.

Exercising eyes result in excellent benefits such as strengthening the eye muscles, to improve the focus of eyes, improve the flexibility of eye movements, and also help in stimulating the vision center of the brain. For mild vision impairments, a series of progressive therapeutic exercises might be helpful in rebuilding the eye muscles and to restore the proper vision. Eye exercises prescribed by the physicians are customized for each and every patient. The age of the patient and their existing eye problems are taken into account before prescribing these exercises. The types of eye exercises are those that include shifting the focus of eyes from nearby objects to farther objects and vice-versa, once the vision is clear, switching the distances, seeing objects in one eye with the another one closed, changing vision to 
different types of objects instead of fixed gaze, fixing the gaze and focussing the vision on a single, solid object and exercising the eye muscles by making certain patterns of movement. The physiological effects of eye strain are Headaches or Migraines, Fatigue, Vertigo and Dizziness, Pain in the Eye, Red, Watery Eyes, Blurred or Double Vision, Dry eyes that feel scratchy, Burning sensation while closing eyes, Neck aches, Back aches, Muscle spasm in the muscles surrounding the eyes and Twitch in the eyelid.

It is a common perception that eye exercises consume a larger time. These eye exercises take a maximum of fifteen to twenty minutes per day which can be allotted by common people. Figure 1 shows the simple pattern of eyelid movements that can be done regularly to improve the flexibility of eye muscles. But this seems to be a very difficult task given the hectic schedule of people. Initially, they show enthusiasm but in the long term lose focus to practise it. Also, children need to be monitored while doing the eye exercises. The exercises which take only few minutes are left off due to want of time. The exercise regime could be made effective and interesting by providing it on their desk monitors. The objectives of this research work are:

1. To develop a LabVIEW program to create an effective eye exercise application.

2. To create an acquisition system to capture the movements of the eye.

3. To design an algorithm to track the movements of the pupil.

4. To design an algorithm to compare these eye movements with the standard pattern and give a match score.

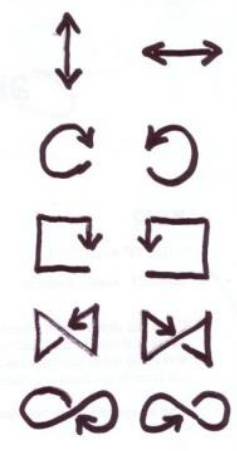

Fig. 1 Recommended patterns for eye movement

\section{Pattern generation and display}

\section{Pattern Generation}

The standard patterns are generated using paint and windows movie maker. A ball is designed using paint and by adding it to movie maker, a continuous display is created. The movement and timings are controllable. Figure 2 shows the computer generated images of the patterns.

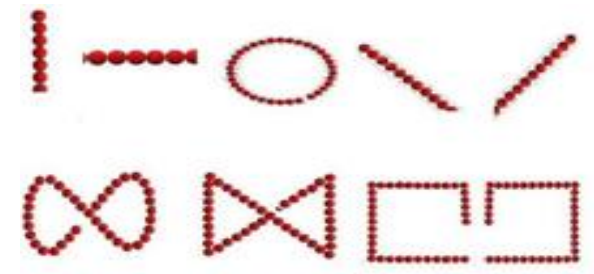

Fig. 2 Proposed Computer Generated patterns

The steps involved are given as follows.

1. Creating the patterns for the exercise which defines the movements using paint as shown in the fig 1 . 
2. Importing the clips which were created using paint to the windows movie maker.

3. Dropping the clips on the story board.

4. Setting timeline according to the movement.

5. Using video effects to assign the movement speed.

6. Finally all the exercises patterns are generated as a single ball movement video. The duration of the video is 40 seconds. Figure 3 presents the screenshots of the steps involved in the pattern generation.

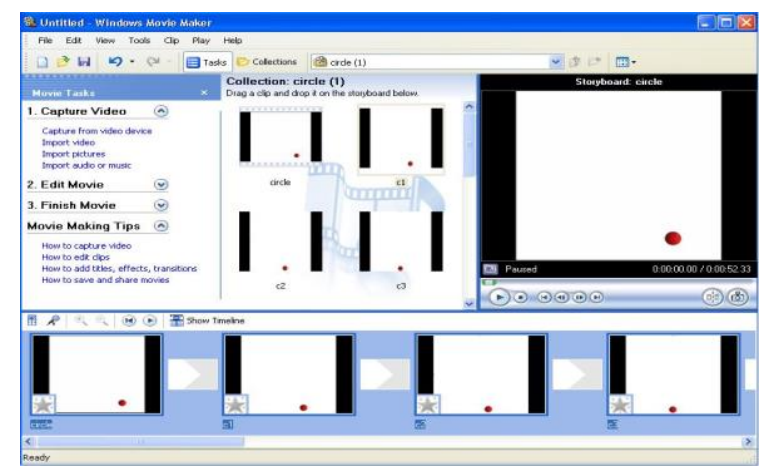

Fig. 3 Screenshot of Windows Movie Maker -Creating Module

\section{Display}

A LabVIEW based virtual instrument is designed to display the pattern on screen and to acquire video from the user. ActiveX controls are employed to embed the pattern video into the LabVIEW program. LabVIEW is an ActiveX container and can house ActiveX controls.

Figure 4 shows the graphical programming involved in connecting the moviemaker file with the VI developed. This VI automatically plays the .avi file specified by the path file in file dialog. This VI is selected as the sub VI in the eye catcher to show the ball movement video, web camera and gaze plot graph simultaneously.

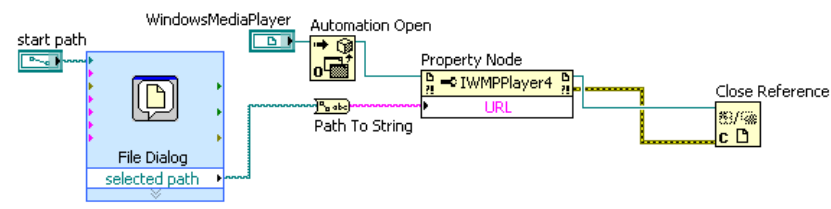

Fig. 4 Pattern deployment using ActiveX

The algorithm is contained inside a single script file. This script file encompasses the functions used in processing the images and its relevant parameters. The algorithm was prototyped using the Vision Assistant feature. Further, the LabVIEW VI Creation Wizard was utilized to create a LabVIEW VI. This Virtual Instrument (VI) is the actual module that runs the prototype that was created through Vision Assistant. The script file of the eye catcher consists of four main functions as pictorially depicted in Figure 5. 


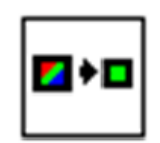

COLOR PLANE EXTRACTION

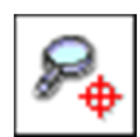

PATTERN MATCHING

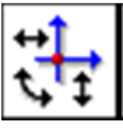

COORDINATE SYSTEM

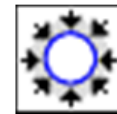

FIND CIRCULAR EDGE

Fig 5. Components of script file of the Eye Catcher

The first component is the process of extracting color planes in which the Red-Green-Blue (RGB), HueSaturation-Value (HSV), or Hue-Saturation-Lightness (HSL) can be extracted. For our application, we extracted the red plane of RGB. The color extracted image is easy for defining the template and for customization for all users. The output of this stage is given for pattern matching step.

Pattern matching - In the context of image processing, edges and patterns are considered to be valuable information bearers. The fiducials and describing features of an image can be located with a high accuracy using this method. From these location measurements, the information such as length of an object, angle of folds/bends and other objective measurements can be made in a robust manner.

Coordinate System - The term coordinate system refers to a group of Virtual Instrumentations (VIs). These VIs identify the coordinates associated with an object in an image. The detection of coordinates is carried out by using the edges that were detected previously or based on the patterns identified earlier. In this work, we have used the horizontal and vertical motion coordinate system modes for finding the $\mathrm{x}$ and $y$ values of the centre of the pattern matching step.

IMAQ Find Circular Edge - The IMAQ module is used to locate the points of intersection between a set of search lines within a circular area, or annulus, and then finds the best fit circle. The inward circle option is chosen. The output of this step gives the best fit circle in terms of the centre and the radius of the circle.

\section{Acquisition Of Eye Movement Images}

For effective eye tracking, a web camera is employed which should be placed on direct line of contact with the eye of the user. When the video was acquired, a hurdle encountered was the change of pupil color in different persons. This would make the processing difficult. Also, a web camera needs spot illumination on the face of the user to extract meaningful images. This situation demands an alternate way to video acquisition.

To eliminate the shortcomings, the choice of an IR camera is looked into. An IR camera is used to capture the eye movements of the user which is transferred to the computer through real time in-line processing. A common camera forms an image using visible light, whereas, an IR camera forms an image using infra red radiation at wavelength upto $14000 \mathrm{~nm}$. The infra red radiations are beyond the vision of human eye, but the sensors integrated into the camera sensors are able to detect those rays and generate black and white images that are of high quality.

An ordinary PC based web camera is dismantled such that by modifying, it can be used as an IR camera for capturing the eye movements. An IR filter is present in all web cameras in order to filter out the IR rays and allow only visible light so that they are in RGB plane. To obtain IR images, the IR filter is removed from the casing at the rear end of the lens. Thus it allows the transmission of IR rays through 
the lens to form images. Figure 5 is a photograph of the camera lens after removal of IR filter. IR LEDs are externally used to support IR radiations coming out of the eye. Power supply to the LEDs is provided from the camera chip. Hence, no external power supply is required which reduces the bulky nature of the capture module. The lights from IR LEDs are placed in such a manner that it transmits light to the eye which when captured gives valuable information about the pupil. Figure 6 shows the front view of the module developed to capture the IR images.

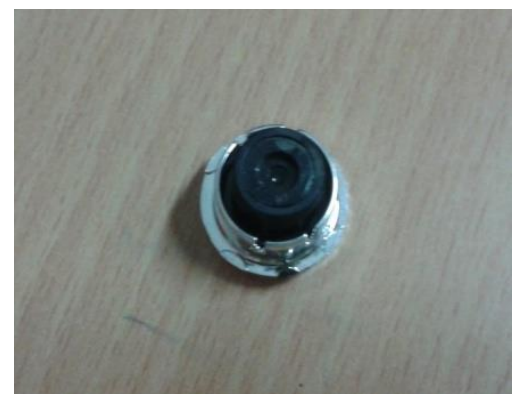

Fig. 5 Infra Red Camera used to capture eye movements

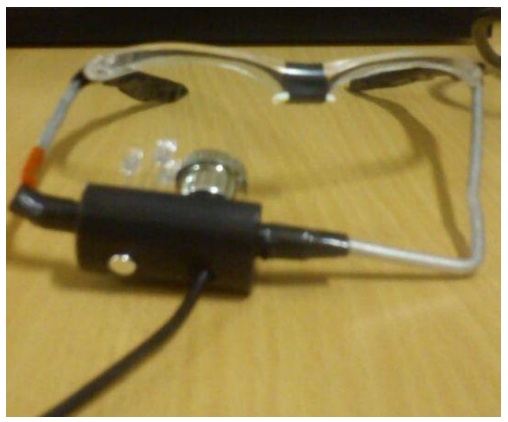

Fig. 6 Front view of the capture module

\section{Development of graphical programming module}

The installed camera is connected to the PC via a USB link. The same LabVIEW module is now made to acquire the real time streaming video from the camera. Vision acquisition module is used to create and edit the acquisition using the vision acquisition express VI. The NI vision acquisition wizard is launched by placing the Express VI in the block diagram. The NI Vision Acquisition Wizard consists of four main sections: Acquisition source, Acquisition type, Acquisition settings and selection of controls and indicators.

Vision Assistant Module is next employed to prototype the developed image processing application. By using the vision assistant scripting feature, custom image processing applications can be designed. The algorithm is recorded in a script file, which contains the processing functions and relevant parameters. The script file of the eye catcher consists of four main functions: Color plane extraction, Pattern matching, Coordinate system and Finding circular edge.

After setting the required steps in vision express modules (vision acquisition and vision assistant), both are made to function under a while loop. This step continuously acquires the images from the USB 
webcam and tracks the pupil. The best fitted circle contains the information about the $\mathrm{x}$ and $\mathrm{y}$ co ordinates of the centre of the pupil. The values are separated and plotted in the x-y graph. This gives the gaze plot i.e., the direction at which the eye moves.

\section{Algorithm For Eye Tracking}

A novel algorithm for detecting eye contours from face images is proposed in [1]. The regions of the eye are assumed to be known priory in a naïve manner. The algorithm employed has several steps. In the first step, the radius of the iris is measured by detecting the centre of the iris region. Based on identifying the luminance valley points, the contour of the upper eyelid is traced by the algorithm. In [2] proposed by Zhonglong et al., algorithm to extract eye features, including pupil center and radius, eye corners and eyelid contours, from frontal face images. The method detects and estimates pupil center in $\mathrm{H}$ channel of HSV color space, and then pupil radius is estimated and refined. In [3], Ghassan J. Mohammed, et al., proposed a new algorithm based on the angular integral projection function (AIPF) to localize eyeball (iris outer boundary) in iris images. The proposed algorithm adopts boundary points detection and curve fitting within gray level images. An eye tracking device that records consensual reflex was reported [4], in which one eye was illuminated with Near Infra Red (NIR) and the other eye was illuminated with visible-light pulse. Under this arrangement, the images of the pupil of the eyes were captured. The features of the iris that were extracted from the images were termed as dynamic features. In the research work [5], the method of Angular Integral Projection Function (AIPF), was employed to find the approximate pupil centre, to detect a set of pupil's radial boundary points, and a circle to the detected boundary points was fitted. In the work proposed by Cuong et al.[6], the image was binarized with a dynamic threshold. The geometry features of the eye image were extracted from binary image. The center of iris was detected by matching between an iris boundary model and image contours.

In the paper [7] proposed by Ann A. Jarjes et. al.,the precise pupil boundary and localized limbic boundary were detected based on AIPF and a GVF snake algorithm. In [8], an algorithm is proposed by Zhifei Xu and Pengfei Shi.,to precisely estimate pupil features. First, a pupil parameters estimation step is taken and a weight ring mask is created. Then, a weighted Hough transform is used to precisely extract pupil features. A template for correlating various regions of the facial image was proposed [9]. Since eyes are a prominent part of the face, the area of the image that was highly correlated with the template was identified as the eye region. A proposal for an eye contour extraction method was given by [10] which combine a simplified version of Active Shape Model (ASM) with a gradient method used to extract the eyelids and a simple method to extract iris boundary. Paper [11] proposed an algorithm to simultaneously detect the blink rate for click of the mouse and pupil localization using edge analysis for cursor movement. In the research work[13], the effect of variation in lighting conditions and varied backgrounds were explored. It was observed that the algorithms performed significantly different for the conditions mentioned above and for color images. The results of this work showed that the algorithm was able to detect skin regions from the entire image through a novel compensation technique for lighting differences. Futher, through methods of nonlinear color transformation, the face candidates were recognized based on the spatial arrangement of these skin patches.

Mohammad H. Mahoor et al. [14] proposed an improved Active Shape Model (ASM) to extract features of face from the images. The improvements were made primarily on the identification of mouth and eyes, using RGB information to represent the feature points and utilizing affine transformation to compensate for head pose variations. The centre of the mouth and eyes were identified using the color information from the images. The feature points had localized structures that were revealed by the RGB values. Variations in the head pose create changes to the facial features. 2D affine transformation has been shown to be effective in aligning the facial features with the Active Shape Model.

Detection of face from frontal color images was done using a hybrid technique [15] that combined facial feature extraction and Principal Component Analysis (PCA). The primary facial features such as eyes and mouth were automatically detected by the Recursive Shortest Spanning Tree (RSST). This 
algorithm took into account the properties of the associated image regions, that were extracted through color segmentation. In the work proposed by Athanasios Nikolaidiset et.al, [16], the aim of the work was to provide a framework to decisively identify a set of features that could help in face recognition and face tracking systems. This work utilized an improved version of adaptive Hough transform. This improved method was used to detect curves in the images, identifying the minima among the candidates to be considered as candidates. This method also enabled localization of inner facial features, through which inner face contours were modelled as active models. The determination of pose was also done through properties of projective geometry. In another research work [17], the focus was on the color segmentation that is an important pre-processing step in the face detection methods. In this paper effective face color segmentation in the HSI (Hue, Saturation and Intensity) space is proposed which is to modify the merging algorithm of the catchment basins obtained by the watershed segmentation method by adding a criterion based on the relevance of the Hue component. The work reported by [18] is directed on the design of objective metrics measured from the. This work explored the addition of visual attention while measuring the features of the image. While many works have been reported on developing computational models for visual attention, this work was focussed on conducting experiments to track the eye movements in order to create ground truths. The results of the experiment have shown that dependability on the attention mode can be reduced due to the reliability of the ground truth generated due to tracking of eye movements.

A publicly available database was reported [19], consisting of experimental eye-tracking data collected through standard video sequences. These sequences find applications in various processes such as video compression, processing, and transmission simulations. In this database, a unique representation of eye-tracking sequences of first and second viewing presented to explore and perform further research. Michal Kowalik proposed the design for the low cost eye tracking glasses for the head mounted systems [20]. The glasses are designed to work with algorithms in the infrared range (dark pupil). Thus environment will not be an hindering factor to track pupil. In a research work reported by Jason S.Babcock et al.[21], the usage of video-based cameras is proposed that are able to monitor both the eye movement and the scene. This was made possible due to the tiny micro-lens video cameras. In many research works, algorithms to detect pupil dynamics were developed and were also implemented through hardware means. This simplifies the complexity involved in real-time eye tracking. However, the trends show that real-time eye tracking can be efficiently accomplished through software alone. In a work by Qiang et. al. [22], a system is developed wherein the level of vigilance of the driver of the vehicle is monitored through the eye movements. The system is developed in such a way to alert the driver in case of threatening driving conditions such as fatigue or dizziness. This system has the potential to prevent accidents.

The works of [23] and [24] deal with practical modifications of the Circular Hough Transform and are presented in order to improve the sensitivity and specificity of the segmentation of medical microscopic images and improve the detection of low contrast objects. An implementation of such a technique showed the identification of a circular arc. The parameters of the arc such as its center point, radius of the arc, length of the arc and its midpoint were estimated from the algorithm. The center of the arc and its radius are primarily used by the Hough transform to detect circular arcs. A peak value in the parameter space of the Hough transform indicates the existence of a circular arc. Hamamatsu et al. in his paper [25] finds the position of the pupil using two different light sources. The detected pupil position in the difference image is utilized together with the glint (corneal reflection light of an infrared light source) position for eye-gaze position determination. This image differentiator made real-time pupil possible, by applying the pupil detector including the noise reducer, which had been already developed. Danjie Zhu et al. in [26] utilises the curvature algorithm to determine the centre and the radius of the pupil exploring the curvature characteristics of the pupil boundary. The curvature value was calculated for each of the boundary point. 
The modified Circular Hough Transform is employed in this work to detect centre of eye during real time processing. The Hough transform finds wide application in image processing techniques due to its ability to identify patterns of a particular shape from the images. It can be technically defined as transferring a Cartesian coordinate $(\mathrm{x}, \mathrm{y})$ to a Polar coordinate $(\mathrm{r}, \theta)$. The object on interest in the image processing context defines the parameter space. In linear algebra, an equation of the form $y=a x+b$ denotes a straight line that passes through a pair of Cartesian coordinates. The values of $a$ and $b$ are the slope and intercept of the line, respectively. However, in the Hough transform, the lines perpendicular to the $\mathrm{x}$-axis are considered to have a value of infinity. Due to this, the parameter space consisting of a and $b$ will be considered to be of infinite size. Hence, the straight line is represented by its normal that is perpendicular to the line of interest. This normal can be represented by an angle $\theta$ and its length can be represented by the equation 1 .

$$
\text { Length of normal }=\mathrm{x} \cos (\theta)+\mathrm{y} \sin (\theta)
$$

In order to detect circular shapes in an image using Circular Hough Transform, the first step is to detect all the edges in the image. At every edge point, a circle is drawn by the algorithm. The $\mathrm{x}$-axis value of the circle is a and the $y$ - axis value is $b$. The radius of the circle is represented by $z$ value. An accumulator will contain numbers corresponding to the number of circles passing through the individual coordinates. Thus the highest numbers correspond to the centre of the circles in the image.

\section{Results And Discussion}

Figure 5 depicts the image frames obtained from the video of the IR camera. The different positions of eyeball movements are captured and tracked using the circular Hough transform.

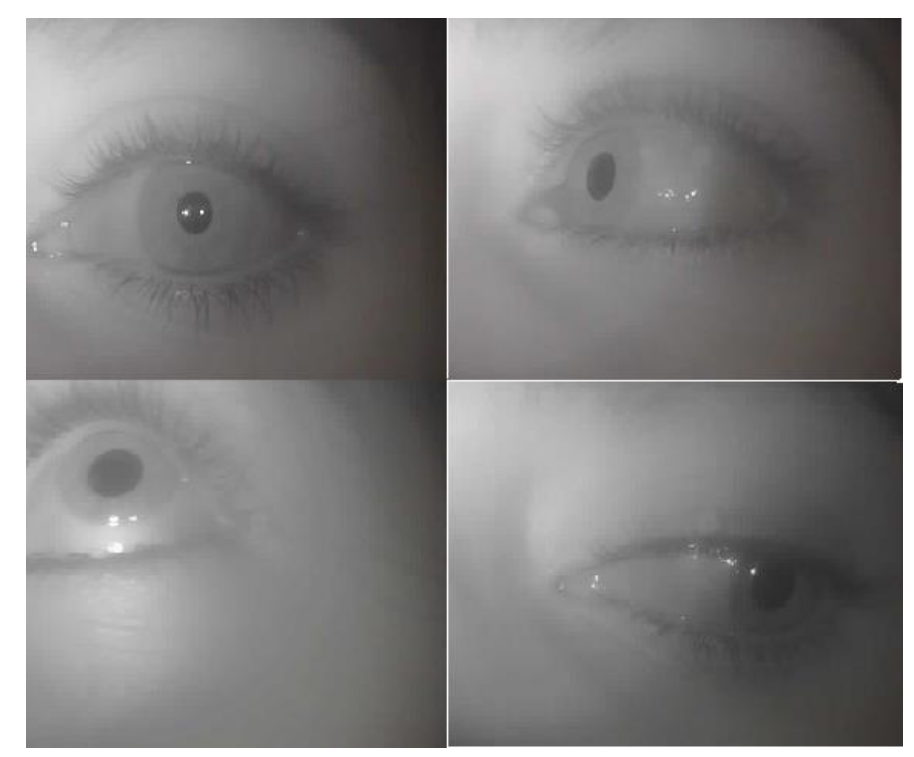

Fig. 5 Video Images from IR camera

The real time eye movements thus captured from the constructed IR camera are processed using LabVIEW environment. Fig. 6 shows the pupil detection done using the algorithm and the corresponding

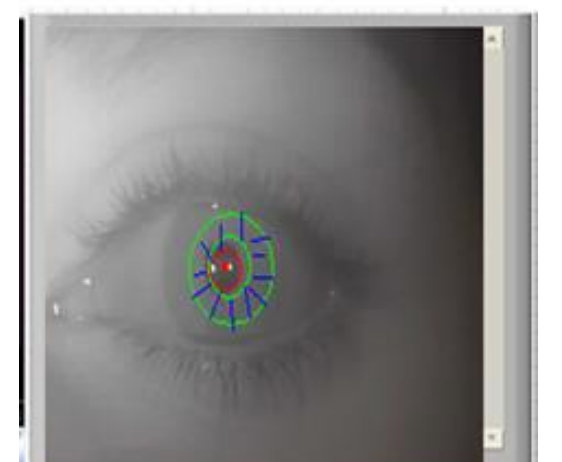


eye movement patterns.

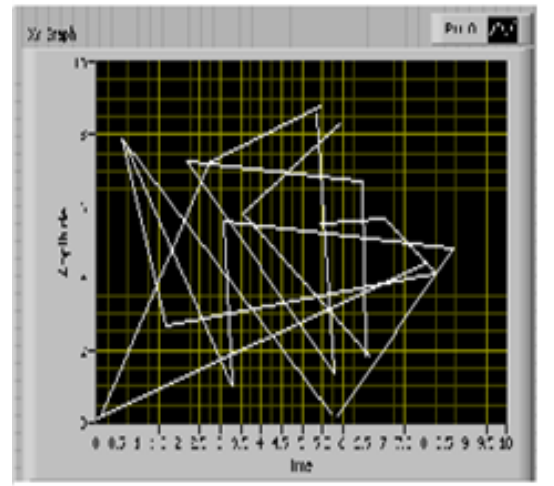

Fig. 6 LabVIEW front panel display showing the tracked eye movement and gaze pattern.

A plot of eyeball movement with respect to time and distance is plotted on the front panel of the VI along with the displayed pattern.

\section{Conclusion}

This work aims to address a noble cause of creating awareness on eye exercises. An eye exercise pattern is displayed on the screen for the user. The users eyeball movements are tracked to compare with the animated pattern. An outline of the process involved is presented in this paper. The system still needs to be refined to create maximum accuracy and benefits; nevertheless, the effort gave a convincing result which may help in developing the idea on a larger scale.

\section{References}

[1] Vladimir Vezhnevets _ Anna Degtiareva, "Robust and accurate eye contour extraction" , International Conference Graphicon,2003.

[2] Zhonglong Zheng, Jie Yang, Limin Yang, "A robust method for eye features extraction on color image”, Pattern Recognition Letters 26, 2005 ,pp:2252-2261.

[3] Ghassan J. Mohammed, Bingrong Hong and Ann A. Jarjes , "eyeball localization based on angular integral projection function “,Journal on Informatica 33, 2009 pp 475-480. 
[4] Ronaldo Martins da Costa and Adilson Gonzaga, "Dynamic features for iris recognition", IEEE Transactions On Systems, Man And Cybernetics-Part B: Cybernetics, Vol. 42,issue-4 , 2012. Pp 1072-1080

[5] Ghassan J. Mohammed, Bing-Rong Hong, Ann A. Jarjes, “Accurate pupil features extraction based on new projection function"., Journal on Computing and Informatics, 2010, Vol. 29, , pp 663-680.

[6] Cuong N.H, Hoang H.T, "Eye-gaze detection with a single webcam based on geometry features extraction", 11th International Conference on Control, Automation, Robotics and Vision, ICARCV , 2010, pp 2507-2512

[7] Ann A. Jarjes, Kuanquan Wang and Ghassan J. Mohammed ,"A new iris segmentation method based on improved snake model and angular integral projection", Research Journal of Applied Sciences, Engineering and Technology 2011, Vol 3(6), pp 558-568.

[8] Zhifei Xu and Pengfei Shi, "A robust and accurate method for pupil features extraction", Pattern Recognition Letters 26, 2005, pp 2252-2261.

[9] Nilamani Bhoi, Mihir Narayan Mohanty, "Template matching based eye detection in facial image" International Journal of Computer Applications 2010 ,Volume 12- No.5, pp 0975 - 8887.

[10] Ke Sun and Hong Wang., "A composite method to extract eye contour". 2005, ACII 2005, LNCS 3784, pp. 112-118.

[11] Michal Ciesla*, Przemyslaw Koziol., "Eye pupil location using webcam”, Journal of Reymonta Vol 4, pp 30-59.

[12] Ann A Jarjes, Kuanquan Wang and Ghassan J. Mohammed, "GVF snake - based method for accurate pupil contour detection”., Information Technology Journal, 2010, Vol 9 , issue 8, pp 16531668

[13] Rein-Lien Hsuy, Mohamed Abdel-Mottalebz "Face detection in color images", IEEE Transactions on Pattern Analysis and Machine Intelligence, 2002, Vol 24,issue 5,pp 696 - 706.

[14] Mohammad H. Mahoor, Mohamed Abdel-Mottaleb, and A-Nasser Ansari, "Active shape model (ASM) for facial feature extraction", 7th International Conference on Automatic Face and Gesture Recognition, 2004 pp. - 148

[15] Ming-Hsuan Yang, David J. Kriegman and Narendra Ahuja," Facial feature extraction and principal component analysis for face detection in color images:, IEEE Transactions on Pattern Analysis and Machine Intelligence, vol 24, no. 1, pp. 696-706, January 2002.

[16] Athanasios Nikolaidis, Ioannis Pitas, "Facial feature extraction and pose determination", Journal on Pattern Recognition 2000,vol 33, pp 1783-1791.

[17] Souhila Guerfi, Jean-Pierre Gambotto, "Implementation of the watershed method in the HSI color space for the face extraction". IEEE Conference On Advanced Video and signal based Survillence, 2005, pp 282-286

[18] Hantao Liu, and Ingrid Heynderickx, "Visual attention in objective image quality assessment: based on eye-tracking data." IEEE transactions on circuits and systems for video technology, 2011, vol. 21 , no. 7 ,

[19] Hadi Hadizadeh, , Mario J. Enriquez, and Ivan V. Bajic, "Eye-tracking database for a set of standard video sequences", IEEE Transcations on Image Processing,2012, Volume: 21 ,Issue:2 ,pp 898 - 903

[20] Michał Kowalik, 'How to build low cost eye tracking glasses for head mounted system', The West Pomeranian University of Technology, Szczecin Poland September 2010.

[21] Jason S.Babcock and Jeff B. Pelz, 'Building a lightweight eye tracking headgear ', Eye Tracking Research \& Application, Texas, 2004.

[22] Qiang Ji1 and Xiaojie Yang, 'Real-Time Eye, Gaze, and Face Pose Tracking for Monitoring Driver Vigilance', Elsevier Science Ltd, 2002.

[23] Marcin Smereka and Ignacy Dule, Ba ,'Circular Object Detection Using A Modified Hough Transform', Int. J. Appl. Math. Comput. Sci., 2008, Vol. 18, No. 1, 85-91.

[24] Soo-Chang Pei and Ji-Hwei Horng, 'Circular arc detection based on Hough transform', Elsevier, Pattern Recognition Letters 16, 1995Hamamatsu, Shizuoka and Yoshinobu Ebisawa, 'Unconstrained pupil detection technique using two light sources and the image difference method', Shizuoka University, Johoku 3-5-1, 432 Japan. 
[25] Danjie Zhu, Steven T. Moore and Theodore Raphan , 'Robust pupil center detection using a curvature algorithm', ELSEVIER , Computer Methods and Programs in Biomedicine 59 (1999) 145-15 